On the compactness of the attainable sets of control systems with $p$-integrable controls

Emrah Akyar 


\title{
ON THE COMPACTNESS OF THE ATTAINABLE SETS OF CONTROL SYSTEMS WITH $p$-INTEGRABLE CONTROLS
}

\author{
EMRAH AKYAR
}

Received 24 November, 2006

\begin{abstract}
Quasi-linear systems governed by $p$-integrable controls, for $1<p<\infty$ with the constraint $\|u\|_{p} \leq \mu_{0}$ are studied. It is proved that the attainable sets of the system at the instant of time $t$ are compact subsets of $\mathbb{R}^{n}$.
\end{abstract}

2000 Mathematics Subject Classification: 93B03, 34H05

Keywords: control system, integral constraint, attainable set

\section{INTRODUCTION}

In this paper quasi-linear control systems which are nonlinear with respect to phase state vector, linear with respect to control vector and where control inputs are constrained by an integral inequality are studied. It is well known that attainable sets play an important role in control theory. Many problems of optimization, dynamics, game theory can be stated and solved in terms of attainable sets. There is already much research on the topological and geometric properties of attainable sets. Linear systems are well studied and most properties of attainable sets are proved [4,5]. For nonlinear systems, attainable sets are still an object of active study (see, e. g., [1-3, 6-8]).

Consider a control system whose behavior is described by the differential equation

$$
x^{\prime}(t)=f(t, x(t))+B(t, x(t)) u(t), \quad x\left(t_{0}\right) \in X_{0},
$$

where $x \in \mathbb{R}^{n}$ is the $n$-dimensional phase state vector of the system, $u \in \mathbb{R}^{r}$ is the $r$-dimensional control vector, $t \in\left[t_{0}, T\right]\left(t_{0}<T<\infty\right)$ is the time, $f(t, x)$ is an $n$ dimensional vector function, $B(t, x)$ is an $(n \times r)$-dimensional matrix function, and $X_{0} \subset \mathbb{R}^{n}$.

It is assumed that the realizations $u(t), t \in\left[t_{0}, T\right]$, of the control $u$ are restricted by the constraint

$$
\int_{t_{0}}^{T}\|u(t)\|^{p} d t \leq \mu_{0}^{p}, \quad \mu_{0}>0,1<p<\infty
$$

The research was supported by the Anadolu University Research Foundation through Project No. AUAF 051025 . 
where $\|\cdot\|$ denotes the Euclidean norm. It is also assumed that the functions $(t, x) \mapsto$ $f(t, x),(t, x) \mapsto B(t, x)$ and the set $X_{0}$ satisfy the following conditions:

(A) The set $X_{0} \subset \mathbb{R}^{n}$ is compact;

(B) The functions $(t, x) \mapsto f(t, x)$ and $(t, x) \mapsto B(t, x)$ are continuous with respect to $(t, x)$ and locally Lipschitz with respect to $x$, that is, for any bounded set $D \subset\left[t_{0}, T\right] \times \mathbb{R}^{n}$, there exist Lipschitz constants $L_{i}=L_{i}(D) \in(0, \infty)$, $i=1,2$, such that

$$
\begin{aligned}
\left\|f\left(t, x^{*}\right)-f\left(t, x_{*}\right)\right\| & \leq L_{1}\left\|x^{*}-x_{*}\right\|, \\
\left\|B\left(t, x^{*}\right)-B\left(t, x_{*}\right)\right\| & \leq L_{2}\left\|x^{*}-x_{*}\right\|
\end{aligned}
$$

for any $\left(t, x^{*}\right)$ and $\left(t, x_{*}\right)$ from $D$;

(C) There exist constants $\gamma_{i} \in(0, \infty), i=1,2$, such that

$$
\begin{aligned}
\|f(t, x)\| & \leq \gamma_{1}(1+\|x\|), \\
\|B(t, x)\| & \leq \gamma_{2}(1+\|x\|)
\end{aligned}
$$

for every $(t, x) \in\left[t_{0}, T\right] \times \mathbb{R}^{n}$.

Every function $u \in L_{p}\left(\left[t_{0}, T\right], \mathbb{R}^{r}\right), 1<p<\infty$, satisfying inequality (1.2) is said to be an admissible control, where $L_{p}\left(\left[t_{0}, T\right], \mathbb{R}^{r}\right)$ denotes the space of $p$-power integrable functions. By the symbol $\mathcal{U}$ we denote the set of all admissible control functions $u$.

Let $u_{*} \in \mathcal{U}$. An absolutely continuous function $x_{*}:\left[t_{0}, T\right] \rightarrow \mathbb{R}^{n}$ which satisfies the equation

$$
x_{*}^{\prime}(t)=f\left(t, x_{*}(t)\right)+B\left(t, x_{*}(t)\right) u_{*}(t)
$$

a.e. on $\left[t_{0}, T\right]$ and has the property

$$
x_{*}\left(t_{0}\right)=x_{0} \in X_{0}
$$

is said to be a solution of system (1.1) with the initial condition (1.3) generated by the admissible control function $u_{*}$. By the symbol $X\left(t_{0}, x_{0}\right)$, we denote the set of all solutions of system (1.1) with initial condition $x\left(t_{0}\right)=x_{0}$, generated by all admissible control functions $u \in U$. We also put

$$
\begin{gathered}
X\left(t_{0}, X_{0}\right)=\left\{x \in X\left(t_{0}, x_{0}\right): x_{0} \in X_{0}\right\}, \\
X\left(t ; t_{0}, X_{0}\right)=\left\{x(t) \in \mathbb{R}^{n}: x \in X\left(t_{0}, X_{0}\right)\right\} .
\end{gathered}
$$

The set $X\left(t ; t_{0}, X_{0}\right)$ is called the attainable set of the system (1.1) with constraint (1.2) at the instant of time $t$. It is obvious that the set $X\left(t ; t_{0}, X_{0}\right)$ consists of all $x \in \mathbb{R}^{n}$, at which the solutions of the system (1.1) which are generated by all possible controls $u \in \mathcal{U}$ arrive at the instant of time $t \in\left[t_{0}, T\right]$.

In this paper we prove that the attainable set $X\left(t ; t_{0}, X_{0}\right)$ defined above is a compact subset of $\mathbb{R}^{n}$ for all $t \in\left[t_{0}, T\right]$. 


\section{PREliminaries}

First, let us give a useful inequality which will be used frequently in the following sections:

$$
\int_{t_{0}}^{t}\left(K_{1}+K_{2}\|u(\tau)\|\right) d \tau \leq K_{1}\left(T-t_{0}\right)+K_{2}\left(T-t_{0}\right)^{\frac{p-1}{p}} \mu_{0}
$$

for every $u \in \mathcal{U}$ and all $t \in\left[t_{0}, T\right]$, where $K_{1}$ and $K_{2}$ are positive constants. This inequality can be easily obtained from the Hölder integral inequality [9, p. 122].

The following proposition states that the graphs of the all solutions of system (1.1) with constraint (1.2) is bounded.

Proposition 1. The inequality

$$
\|x(t)\| \leq r
$$

holds for all $x \in X\left(t_{0}, X_{0}\right)$ and $t \in\left[t_{0}, T\right]$, where

$$
\begin{aligned}
q & =\gamma_{1}\left(T-t_{0}\right)+\gamma_{2} \mu_{0}\left(T-t_{0}\right)^{\frac{p-1}{p}}, \\
d_{*} & =\max \left\{\|v\|: v \in X_{0}\right\},
\end{aligned}
$$

and

$$
r=\left(d_{*}+q\right) \exp (q) .
$$

Proof. Let $x \in X\left(t_{0}, X_{0}\right)$ be any solution of system (1.1). Then there exist $x_{0} \in X_{0}$ and $u \in U$ such that

$$
x(t)=x_{0}+\int_{t_{0}}^{t}(f(\tau, x(\tau))+B(\tau, x(\tau)) u(\tau)) d \tau, \quad t \in\left[t_{0}, T\right] .
$$

Calculating the norm of both sides, using condition (C), and recalling that $d_{*}$ is given by (2.3), we obtain,

$$
\begin{aligned}
\|x(t)\| \leq d_{*}+\gamma_{1}\left(T-t_{0}\right)+\gamma_{1} \int_{t_{0}}^{t}\|x(\tau)\| d \tau & +\gamma_{2} \int_{t_{0}}^{t}\|u(\tau)\| d \tau \\
& +\gamma_{2} \int_{t_{0}}^{t}\|x(\tau)\|\|u(\tau)\| d \tau .
\end{aligned}
$$

Since $u \in \mathcal{U}$, using Hölder's integral inequality, we find

$$
\int_{t_{0}}^{t}\|u(\tau)\| d \tau \leq\left(\int_{t_{0}}^{t} 1^{\frac{p}{p-1}} d \tau\right)^{\frac{p-1}{p}}\left(\int_{t_{0}}^{t}\|u(\tau)\|^{p} d \tau\right)^{\frac{1}{p}} \leq \mu_{0}\left(T-t_{0}\right)^{\frac{p-1}{p}} .
$$

By virtue of (2.6) and since $q=\gamma_{1}\left(T-t_{0}\right)+\gamma_{2} \mu_{0}\left(T-t_{0}\right)^{\frac{p-1}{p}}$, we obtain

$$
\|x(t)\| \leq d_{*}+q+\int_{t_{0}}^{t}\left(\gamma_{1}+\gamma_{2}\|u(\tau)\|\right)\|x(\tau)\| d \tau .
$$


Using Gronwall's inequality [9, p. 189], we get

$$
\|x(t)\| \leq\left(d_{*}+q\right) \exp \left(\int_{t_{0}}^{t}\left(\gamma_{1}+\gamma_{2}\|u(\tau)\|\right) d \tau\right) .
$$

In view of inequality (2.1), we find

$$
\|x(t)\| \leq\left(d_{*}+q\right) \exp (q) .
$$

The right hand side of this last inequality is exactly the number $r$ appearing in (2.4), so it is clear that the inequality

$$
\|x(t)\| \leq r
$$

holds for all $x \in X\left(t_{0}, X_{0}\right)$ and all $t \in\left[t_{0}, T\right]$.

Definition. The set

$$
Z\left(t_{0}, X_{0}\right)=\left\{(t, x(t)) \in\left[t_{0}, T\right] \times \mathbb{R}^{n}: x \in X\left(t_{0}, X_{0}\right)\right\}
$$

is called the integral funnel of system (1.1) with constraint (1.2).

Proposition 1 yields the following corollaries.

Corollary 1. Graphs of all solutions of system (1.1) are bounded by the cylinder

$$
D=\left\{(t, x) \in\left[t_{0}, T\right] \times \mathbb{R}^{n}:\|x\| \leq r\right\} .
$$

In other words, the inclusion $Z\left(t_{0}, X_{0}\right) \subset D$ holds.

Here, $r>0$ is defined by the formula (2.4). From now on, $D$ will denote the cylinder (2.7).

Corollary 2. The set of all solutions $X\left(t_{0}, X_{0}\right)$ of system (1.1) is a uniformly bounded subset of $C\left(\left[t_{0}, T\right], \mathbb{R}^{n}\right)$. Furthermore, the attainable sets at the instant of time $t$ are bounded subsets of $\mathbb{R}^{n}$ for all $t \in\left[t_{0}, T\right]$.

Here, $C\left(\left[t_{0}, T\right], \mathbb{R}^{n}\right)$ denotes the space of continuous functions $x:\left[t_{0}, T\right] \rightarrow \mathbb{R}^{n}$ with the norm $\|x\|_{C}=\max _{t \in\left[t_{0}, T\right]}\|x(t)\|$.

\section{COMPACTNESS OF ATTAINABLE SETS}

In this section, by means of Ascoli's theorem [9, p. 109], it is proved that the attainable set of the system (1.1) with constraint (1.2) is a compact subset of $\mathbb{R}^{n}$ at the instant of time $t \in\left[t_{0}, T\right]$. Since we have already shown that the set of all solutions is uniformly bounded, we only need to prove its equicontinuity and relative compactness.

The following proposition states that set of all solutions $X\left(t_{0}, X_{0}\right)$ is an equicontinuous family of functions in $C\left(\left[t_{0}, T\right], \mathbb{R}^{n}\right)$.

Proposition 2. The set of all solutions $X\left(t_{0}, X_{0}\right)$ of system (1.1) with constraint (1.2) is an equicontinuous family in $C\left(\left[t_{0}, T\right], \mathbb{R}^{n}\right)$. 
Proof. Let $\varepsilon>0$ and $x \in X\left(t_{0}, X_{0}\right)$ be any solution of system (1.1). Then there exist $x_{0} \in X_{0}$ and $u \in U$ such that the equality

$$
x(t)=x_{0}+\int_{t_{0}}^{t}(f(\tau, x(\tau))+B(\tau, x(\tau)) u(\tau)) d \tau
$$

holds for all $t \in\left[t_{0}, T\right]$.

Let us choose arbitrary $t_{1}, t_{2} \in\left[t_{0}, T\right]$. Without loss of generality we can suppose that $t_{1} \leq t_{2}$. Therefore,

$$
\begin{aligned}
\left\|x\left(t_{1}\right)-x\left(t_{2}\right)\right\| & =\left\|\int_{t_{1}}^{t_{2}}[f(\tau, x(\tau))+B(\tau, x(\tau)) u(\tau)] d \tau\right\| \\
& \leq \int_{t_{1}}^{t_{2}}\|f(\tau, x(\tau))\| d \tau+\int_{t_{1}}^{t_{2}}\|B(\tau, x(\tau))\|\|u(\tau)\| d \tau .
\end{aligned}
$$

Since the functions $f$ and $B$ are continuous and the set $D$ is compact, we can set

$$
\begin{aligned}
& K_{1}=\max \{\|f(t, x)\|:(t, x) \in D\}, \\
& K_{2}=\max \{\|B(t, x)\|:(t, x) \in D\} .
\end{aligned}
$$

Then we obtain

$$
\left\|x\left(t_{1}\right)-x\left(t_{2}\right)\right\| \leq K_{1}\left|t_{2}-t_{1}\right|+K_{2} \int_{t_{1}}^{t_{2}}\|u(\tau)\| d \tau .
$$

Using Hölder's integral inequality we get

$$
\begin{aligned}
\left\|x\left(t_{1}\right)-x\left(t_{2}\right)\right\| & \leq K_{1}\left|t_{2}-t_{1}\right|+K_{2}\left(\int_{t_{1}}^{t_{2}} 1^{\frac{p}{p-1}} d \tau\right)^{\frac{p-1}{p}}\left(\int_{t_{1}}^{t_{2}}\|u(\tau)\|^{p} d \tau\right)^{\frac{1}{p}} \\
& \leq K_{1}\left|t_{2}-t_{1}\right|+K_{2} \mu_{0}\left|t_{2}-t_{1}\right|^{\frac{p-1}{p}}
\end{aligned}
$$

for all $t_{1}, t_{2} \in\left[t_{0}, T\right]$. Setting $K=\max \left\{K_{1}, \mu_{0} K_{2}\right\}$, we get

$$
\begin{aligned}
& \left\|x\left(t_{1}\right)-x\left(t_{2}\right)\right\| \leq K\left|t_{2}-t_{1}\right|+K\left|t_{2}-t_{1}\right|^{\frac{p-1}{p}} \\
& \quad=K\left|t_{2}-t_{1}\right|^{\frac{p-1}{p}}\left(1+\left|t_{2}-t_{1}\right|^{\frac{1}{p}}\right) \leq K\left(1+\left(T-t_{0}\right)^{\frac{1}{p}}\right)\left|t_{2}-t_{1}\right|^{\frac{p-1}{p}}
\end{aligned}
$$

for all $t_{1}, t_{2} \in\left[t_{0}, T\right]$. Setting $K_{*}=K\left(1+\left(T-t_{0}\right)^{\frac{1}{p}}\right)$, we find

$$
\left\|x\left(t_{1}\right)-x\left(t_{2}\right)\right\| \leq K_{*}\left|t_{2}-t_{1}\right|^{\frac{p-1}{p}} .
$$

Therefore, if $t_{1}$ and $t_{2}$ are such that $\left|t_{1}-t_{2}\right|<\delta(\varepsilon)$ with $\delta(\varepsilon)=\left(\varepsilon / K_{*}\right)^{\frac{p}{p-1}}$, then the inequality

$$
\left\|x\left(t_{1}\right)-x\left(t_{2}\right)\right\|<\varepsilon
$$

holds. Since the element $x \in X\left(t_{0}, X_{0}\right)$ is arbitrary, this completes the proof of our proposition. 
Combining Corollary 2, Proposition 2, and Ascoli's theorem, we obtain the following corollary.

Corollary 3. The set of all solutions $X\left(t_{0}, X_{0}\right)$ of system (1.1) with constraint (1.2) is a relatively compact subset of $C\left(\left[t_{0}, T\right], \mathbb{R}^{n}\right)$. $^{*}$

Proposition 3. The set of all solutions $X\left(t_{0}, X_{0}\right)$ of system (1.1) with constraint (1.2) is a closed subset of $C\left(\left[t_{0}, T\right], \mathbb{R}^{n}\right)$.

Proof. Let $x_{k}$ be any sequence in $X\left(t_{0}, X_{0}\right)$ and $x_{k} \rightarrow x_{*}$ as $k \rightarrow \infty$. It is necessary to prove that $x_{*} \in X\left(t_{0}, X_{0}\right)$.

Since $x_{k} \in X\left(t_{0}, X_{0}\right)$ for all $k=1,2, \ldots$, there exist $c_{k} \in X_{0}$ and $u_{k} \in U$ such that

$$
x_{k}(t)=c_{k}+\int_{t_{0}}^{t}\left(f\left(\tau, x_{k}(\tau)\right)+B\left(\tau, x_{k}(\tau)\right) u_{k}(\tau)\right) d \tau
$$

holds for all $t \in\left[t_{0}, T\right]$. Since $X_{0} \subset \mathbb{R}^{n}$ is compact, the sequence $\left\{c_{k}\right\}_{k=1}^{\infty}$ contains a convergent subsequence. Assume without loss of generality that $c_{k} \rightarrow c_{0} \in X_{0}$ as $k \rightarrow \infty$.

In view of the fact that the set of admissible control functions

$$
\mathcal{U}=\left\{u \in L_{p}\left(\left[t_{0}, T\right], \mathbb{R}^{r}\right): \int_{t_{0}}^{T}\|u(\tau)\|^{p} d \tau \leq \mu_{0}^{p}\right\},
$$

where $\mu_{0}>0$ and $p \in(1, \infty)$, is a weakly compact subset of $L_{p}\left(\left[t_{0}, T\right], \mathbb{R}^{r}\right)$ and $u_{k} \in$ $U$ for all $k=1,2, \ldots$, the sequence $\left\{u_{k}\right\}_{\substack{w=1 \\ w}}^{\infty}$ has weakly convergent subsequence. Suppose without loss of generality that $u_{k} \stackrel{\underset{w}{\rightarrow}}{\rightarrow} u_{0} \in U$ as $k \rightarrow \infty$.

By the definition of weak convergence, we have

$$
\int_{t_{0}}^{T} v(\tau) u_{k}(\tau) d \tau \rightarrow \int_{t_{0}}^{T} \nu(\tau) u_{0}(\tau) d \tau
$$

as $k \rightarrow \infty$ for all $v \in L_{q}\left(\left[t_{0}, T\right], \mathbb{R}^{r}\right)$, where $p^{-1}+q^{-1}=1$.

Let us denote by $x_{0}$ the solution of system (1.1) generated by admissible control $u_{0} \in \mathcal{U}$ which starts from the initial point $\left(t_{0}, c_{0}\right)$. Then, for all $t \in\left[t_{0}, T\right]$, the value $x_{0}(t)$ can be represented in the form

$$
x_{0}(t)=c_{0}+\int_{t_{0}}^{t}\left(f\left(\tau, x_{0}(\tau)\right)+B\left(\tau, x_{0}(\tau)\right) u_{0}(\tau)\right) d \tau .
$$

${ }^{*}$ That is, $X\left(t_{0}, X_{0}\right)$ is a subset of $C\left(\left[t_{0}, T\right], \mathbb{R}^{n}\right)$ with compact closure. 
From here and condition (B) we obtain

$$
\begin{aligned}
\left\|x_{k}(t)-x_{0}(t)\right\| \leq & \left\|c_{k}-c_{0}\right\|+\int_{t_{0}}^{t}\left\|f\left(\tau, x_{k}(\tau)\right)-f\left(\tau, x_{0}(\tau)\right)\right\| d \tau \\
& +\left\|\int_{t_{0}}^{t}\left[B\left(\tau, x_{k}(\tau)\right) u_{k}(\tau)-B\left(\tau, x_{0}(\tau)\right) u_{0}(\tau)\right] d \tau\right\| \\
\leq & \left\|c_{k}-c_{0}\right\|+L_{1} \int_{t_{0}}^{t}\left\|x_{k}(\tau)-x_{0}(\tau)\right\| d \tau+ \\
& +L_{2} \int_{t_{0}}^{t}\left\|x_{k}(\tau)-x_{0}(\tau)\right\|\left\|u_{k}(\tau)\right\| d \tau+ \\
& +\left\|\int_{t_{0}}^{t} B\left(\tau, x_{0}(\tau)\right)\left(u_{k}(\tau)-u_{0}(\tau)\right) d \tau\right\|
\end{aligned}
$$

for all $k=1,2, \ldots$ and $t \in\left[t_{0}, T\right]$. Since the function $B\left(\cdot, x_{0}(\cdot)\right):\left[t_{0}, T\right] \rightarrow \mathbb{R}^{n \times r}$ is continuous and $c_{k} \rightarrow c_{0}, u_{k} \stackrel{w}{\rightarrow} u_{0}$ as $k \rightarrow \infty$, it follows that, for all $\varepsilon>0$, there exists a $K(\varepsilon)>0$ such that the inequality

$$
\left\|c_{k}-c_{0}\right\|+\left\|\int_{t_{0}}^{t} B\left(\tau, x_{0}(\tau)\right)\left(u_{k}(\tau)-u_{0}(\tau)\right) d \tau\right\| \leq \varepsilon, \quad t \in\left[t_{0}, T\right]
$$

is valid for all $k \geq K(\varepsilon)$. Using (3.1), we get

$$
\left\|x_{k}(t)-x_{0}(t)\right\| \leq \int_{t_{0}}^{t}\left(L_{1}+L_{2}\left\|u_{k}(\tau)\right\|\right)\left\|x_{k}(\tau)-x_{0}(\tau)\right\| d \tau+\varepsilon
$$

for all $k \geq K(\varepsilon)$ and $t \in\left[t_{0}, T\right]$. Applying Gronwall's inequality, we find

$$
\left\|x_{k}(t)-x_{0}(t)\right\| \leq \varepsilon \exp \left(\int_{t_{0}}^{t}\left(L_{1}+L_{2}\left\|u_{k}(\tau)\right\|\right) d \tau\right)
$$

for all $k \geq K(\varepsilon)$ and $t \in\left[t_{0}, T\right]$.

Using (2.1), we obtain

$$
\left\|x_{k}(t)-x_{0}(t)\right\| \leq \varepsilon \exp \left(L_{1}\left(T-t_{0}\right)+L_{2} \mu_{0}\left(T-t_{0}\right)^{\frac{p-1}{p}}\right)
$$

for all $k \geq K(\varepsilon)$ and $t \in\left[t_{0}, T\right]$. This means that $x_{k} \rightarrow x_{0}$ as $k \rightarrow \infty$.

Since the limit is unique, wesee that the solution $x_{*}$ must be equal to $x_{0}$. Therefore, $x_{*} \in X\left(t_{0}, X_{0}\right)$, which implies that the set of all solutions $X\left(t_{0}, X_{0}\right)$ of system (1.1) is closed.

Using the closedness of $X\left(t_{0}, X_{0}\right)$, we also obtain

Corollary 4. The attainable set $X\left(t ; t_{0}, X_{0}\right)$ of system (1.1) with constraint (1.2) at the instant of time $t$ is closed subset of $\mathbb{R}^{n}$ for all $t \in\left[t_{0}, T\right]$.

Combining all the results stated above, we arrive at the following main theorem. 
Theorem. The set of all solutions $X\left(t_{0}, X_{0}\right)$ and the attainable set $X\left(t ; t_{0}, X_{0}\right)$, at the instant of time $t$, of system (1.1) with constraint (1.2) are compact subsets of $C\left(\left[t_{0}, T\right], \mathbb{R}^{n}\right)$ and $\mathbb{R}^{n}$, respectively.

\section{REFERENCES}

[1] P. Collins, "Continuity and computability of reachable sets," Theoret. Comput. Sci., vol. 341, no. 1-3, pp. 162-195, 2005.

[2] A. M. Formalsky and A. N. Sirotin, "On the geometric properties of reachable and controllable sets for linear discrete systems," J. Optim. Theory Appl., vol. 122, no. 2, pp. 257-284, 2004.

[3] K. G. Guseinov, O. Ozer, and E. Akyar, "On the continuity properties of the attainable sets of control systems with integral constraints on control," Nonlinear Anal., vol. 56, no. 3, pp. 433-449, 2004.

[4] H. Hermes and J. P. LaSalle, Functional analysis and time optimal control. New York: Academic Press, 1969, mathematics in Science and Engineering, Vol. 56.

[5] E. B. Lee and L. Markus, Foundations of optimal control theory. New York: John Wiley \& Sons Inc., 1967.

[6] H. W. Lou, "On the attainable sets of control systems with $p$-integrable controls," J. Optim. Theory Appl., vol. 123, no. 1, pp. 123-147, 2004.

[7] S. Perrotta, "On the closure of reachable sets for control systems," J. Optim. Theory Appl., vol. 82, no. 3, pp. 555-569, 1994.

[8] M. V. Topunov, "On the convexity of the reachable set of a bilinear controlled system," Prikl. Mat. Mekh., vol. 67, no. 5, pp. 752-758, 2003.

[9] J. Warga, Optimal control of differential and functional equations. New York: Academic Press, 1972.

Author's address

Emrah Akyar

Anadolu University, Department of Mathematics, 26470, Eskisehir, Turkey

E-mail address: eakyar@anadolu.edu.tr 